\title{
Discrete-Time Sliding-Mode Control of Uncertain Systems with Time-Varying Delays via Descriptor Approach
}

\author{
Maode Yan, ${ }^{1,2}$ Aryan Saadat Mehr, ${ }^{3}$ and Yang Shi ${ }^{2}$ \\ ${ }^{1}$ School of Electronic and Control Engineering, Chang'an University, Xi'an 710064, China \\ ${ }^{2}$ Department of Mechanical Engineering, University of Saskatchewan, 57 Campus Drive, Saskatoon, \\ SK, Canada S7N 5A9 \\ ${ }^{3}$ Department of Electrical and Computer Engineering, University of Saskatchewan, 57 Campus Drive, Saskatoon, \\ SK, Canada S7N $5 A 9$
}

Correspondence should be addressed to Aryan Saadat Mehr, aryan.saadat@usask.ca

Received 12 February 2008; Accepted 2 June 2008

Recommended by Pablo Iglesias

\begin{abstract}
This paper considers the problem of robust discrete-time sliding-mode control (DT-SMC) design for a class of uncertain linear systems with time-varying delays. By applying a descriptor model transformation and Moon's inequality for bounding cross terms, a delay-dependent sufficient condition for the existence of stable sliding surface is given in terms of linear matrix inequalities (LMIs). Based on this existence condition, the synthesized sliding mode controller can guarantee the sliding-mode reaching condition of the specified discrete-time sliding surface for all admissible uncertainties and time-varying delays. An illustrative example verifies the effectiveness of the proposed method.
\end{abstract}

Copyright (c) 2008 Maode Yan et al. This is an open access article distributed under the Creative Commons Attribution License, which permits unrestricted use, distribution, and reproduction in any medium, provided the original work is properly cited.

\section{INTRODUCTION}

Time delay often appears in many engineering systems, such as networked control systems, telecommunication systems, nuclear reactors, chemical engineering systems, and so on. Since time delay is often an important source of poor performance and instability, considerable attention has been dedicated to stability analysis and control synthesis for timedelay systems. Many research results have been reported and well documented in $[1,2]$, and the references therein. A linear matrix inequalities (LMIs-) based approach to delaydependent stability for continuous-time uncertain systems with time-varying delays is proposed by using the LeibnizNewton formula in [3]. In [4], a new technique is studied by incorporating both the time-varying-delayed state and the delay-upper-bounded state to make full use of all available information in the design. It is worth noting that the wide use of digital computers in practical control systems requires the stability analysis and controller synthesis for discrete-time time-delay systems. In [5], delay-dependent sufficient conditions for the existence of the guaranteed cost controller in terms of LMIs are derived for uncertain discrete-time systems with constant delays. A necessary and sufficient condition is established for stabilizing a networked control system by modeling both the sensor-to-controller and controller-to-actuator delays as Markov chains in [6]. In [7], Gao et al. propose an output-feedback stabilization method for discrete-time systems with time-varying delays. This method is dependent on the lower and upper delay bounds, and is further extended to discrete-time systems with norm-bounded mismatched uncertainties. Further, a less conservative result on stability of discrete-time systems with time-varying delays, which also incorporates both the time-varying-delayed state and the delay-upper-bounded state, is obtained by defining new Lyapunov functions in [8].

It is well known that the sliding-mode control (SMC) is an effective method to achieve robustness and invariance to matched uncertainties and disturbances on the sliding surface [9]. In recent years, increasing attention has been paid to the design of SMC for uncertain systems with time delay. One approach based on lumped sliding surface has been applied to design an SMC law for state-delay systems in [10]. In [11], the SMC method is proposed for systems with mismatched parametric uncertainties and time delay, and a delayindependent sufficient condition for the existence of linear sliding surface is derived in terms of LMIs; the synthesized 
sliding-mode controller can guarantee the reaching condition of the specified sliding surface. In [12], an observerbased sliding mode control is studied for continuous-time state-delayed systems with unmeasurable states and nonlinear uncertainties, and a sufficient condition of asymptotic stability is derived for the overall closed-loop systems.

Recently, a new descriptor model transformation is introduced for stability analysis [13], controller design [5, 14], and filtering design [15] of time-delay systems. This approach can significantly reduce the overdesign entailed in the existing methods because of the model equivalence and less conservative bound on cross terms [14]. In [16], a Lyapunov-Krasovskii techniques-based delay-dependent descriptor approach to stability and control of linear systems with time-varying delays is proposed, and is further combined with the sliding mode control result to deal with the mismatched uncertainties and unknown nonlinear functions. However, to the best of authors' knowledge, the descriptor approach has not been fully investigated for research on delay-dependent DT-SMC design of linear systems with mismatched uncertainties and time-varying delays. This motivates our research work in this paper.

In this paper, we first propose a delay-dependent sufficient condition for the existence of stable sliding surface via the descriptor approach. Then, based on this existence condition, the synthesized discrete-time sliding-mode controller is designed to guarantee the sliding mode reaching condition of the specified discrete-time sliding surface. The rest of the paper is organized as follows. Section 2 describes the problem formulation and some necessary preliminary results. In Section 3, sufficient conditions for the existence of stable sliding surface are presented in terms of LMIs and a robust DT-SMC law is presented. This DT-SMC law can guarantee the sliding-mode reaching condition of the specified discrete-time sliding surface for all admissible uncertainties and time-varying delays. An illustrative example is used to demonstrate the validity and effectiveness of the proposed method in Section 4. Finally, Section 5 offers some concluding remarks.

Throughout this paper, superscript " $\mathrm{T}$ " stands for matrix transposition, $\mathbb{R}^{n}$ denotes the $n$-dimensional Euclidean space, $\mathbb{R}^{n \times m}$ is the set of all real matrices of dimension $n \times m, P>0$ means that $P$ is real symmetric and positive definite matrix, $I$ and 0 represent identity matrix and zero matrix with appropriate dimensions. In symmetric block matrices or long matrix expressions, we use an asterisk $(*)$ to represent a term that is induced by symmetry. $\operatorname{diag}\{\cdot\}$ stands for a block-diagonal matrix. Matrices, if their dimensions are not explicitly stated, are assumed to be compatible for algebraic operations.

\section{PROBLEM FORMULATION}

Consider the following discrete-time uncertain system with time-varying delays in the regular form:

$$
\begin{aligned}
x_{1}(k+1)= & \left(A_{11}+\Delta A_{11}\right) x_{1}(k)+\left(A_{d 11}+\Delta A_{d 11}\right) x_{1}(k-d(k)) \\
& +\left(A_{12}+\Delta A_{12}\right) x_{2}(k),
\end{aligned}
$$

$$
\begin{gathered}
x_{2}(k+1)=\sum_{i=1}^{2}\left[A_{2 i} x_{i}(k)+A_{d 2 i} x_{i}(k-d(k))\right]+B u(k)+f(k, x(k)), \\
x(k)=\varphi(k), \quad k=-d_{M},-d_{M}+1, \ldots, 0,
\end{gathered}
$$

where $x_{1} \in \mathbb{R}^{n-m}, x_{2} \in \mathbb{R}^{m}, x(k)=\left(x_{1}, x_{2}\right)^{\mathrm{T}}$ is the system state vector, $u(k) \in \mathbb{R}^{m}$ is the control input, $f(k, x(k)) \in \mathbb{R}^{m}$ is an unknown nonlinear function representing the unmodeled dynamics and external disturbances, $d(k)$ is the unknown time-varying delays, $A_{11}, A_{d 11}, A_{12}, A_{21}, A_{22}, A_{d 21}, A_{d 22}$, and $B$ represent real constant system matrices with appropriate dimensions, $\Delta A_{11}, \Delta A_{d 11}$, and $\Delta A_{12}$ are time-varying matrix functions representing parameter uncertainties, $\varphi(k) \in \mathbb{R}^{n}$ represents initial values of $x(k)$. It is assumed that the system uncertainties are norm-bounded with the following form:

$$
\left[\Delta A_{11} \Delta A_{12}\right]=E_{1} F_{1}(k)\left[H_{1} H_{3}\right], \quad \Delta A_{d 11}=E_{2} F_{2}(k) H_{2},
$$

where $E_{1}, E_{2}$, and $H_{i}(i=1,2,3)$ are known constant matrices with compatible dimensions, and the properly dimensioned matrix $F_{i}(k)$ is an unknown and time-varying matrix of uncertain parameters, but norm bounded as $F_{i}^{\mathrm{T}}(k) F_{i}(k) \leq I, i=1,2$. Associated with system (2), we make the following assumptions.

Assumption 1. The time-varying delays $d(k)$ is assumed to satisfy $d_{m} \leq d(k) \leq d_{M}$, where $d_{m}$ and $d_{M}$ are constant positive scalars representing the lower and upper bounds on the time delay, respectively.

Assumption 2. The pair $\left(A_{11}, A_{12}\right)$ in the nominal system of (2) is controllable.

Assumption 3. Nonlinear function $f(k, x(k))$ is unknown but bounded in the sense of the Euclidean norm.

The design procedure of DT-SMC consists of two steps: design of the stable sliding surface and the reaching motion control law. For discrete-time uncertain systems with timevarying delays, the linear sliding surface is chosen as

$$
S(k)=\bar{C} x(k)=[C I] x(k)=C x_{1}(k)+x_{2}(k)=0
$$

with $\bar{C} \in \mathbb{R}^{m \times n}$ and $C \in \mathbb{R}^{m \times(n-m)}$ is a real matrix to be designed. Once sliding surface satisfies the discretetime sliding mode reaching condition, substituting $x_{2}(k)=$ $-C x_{1}(k)$ into the first subsystem of (2), we obtain the following sliding motion:

$$
\begin{aligned}
x_{1}(k+1)= & \left(A_{11}-A_{12} C+\Delta A_{11}-\Delta A_{12} C\right) x_{1}(k) \\
& +\left(A_{d 11}+\Delta A_{d 11}\right) x_{1}(k-d(k)), \\
x_{1}(k)= & \varphi_{1}(k), \quad k=-d_{M},-d_{M}+1, \ldots, 0 .
\end{aligned}
$$

Here, $\varphi_{1}(k) \in \mathbb{R}^{n-m}$ stands for initial values of $x_{1}(k)$. In order to derive our main results, the following two lemmas are necessary. 
Lemma 1 (see [17], Moon's inequality). Assume that a $\in$ $\mathbb{R}^{n_{a}}, b \in \mathbb{R}^{n_{b}}$, and $N \in \mathbb{R}^{n_{a} \times n_{b}}$. Then for any matrices $X \in$ $\mathbb{R}^{n_{a} \times n_{a}}, Y \in \mathbb{R}^{n_{a} \times n_{b}}$, and $Z \in \mathbb{R}^{n_{b} \times n_{b}}$, the following holds:

$$
-2 a^{\mathrm{T}} N b \leq\left[\begin{array}{l}
a \\
b
\end{array}\right]^{\mathrm{T}}\left[\begin{array}{cc}
X & Y-N \\
Y^{\mathrm{T}}-N^{\mathrm{T}} & Z
\end{array}\right]\left[\begin{array}{l}
a \\
b
\end{array}\right],
$$

where

$$
\left[\begin{array}{ll}
X & Y \\
Y & Z
\end{array}\right] \geq 0
$$

Lemma 2 (see [18]). For any matrices $D \in \mathbb{R}^{n \times p}, E \in \mathbb{R}^{p \times n}$, $F \in \mathbb{R}^{p \times p}$ with $F^{\mathrm{T}}(k) F(k) \leq I$ and scalars $\varepsilon>0$,

$$
D F E+E^{\mathrm{T}} F^{\mathrm{T}} D^{\mathrm{T}} \leq \varepsilon^{-1} D D^{\mathrm{T}}+\varepsilon E^{\mathrm{T}} E .
$$

The main objective of this paper is to design the sliding surface $S(k)$ and a DT-SMC law $u(k)$ such that

(1) the sliding surface is asymptotically stable;

(2) the DT-SMC law can guarantee discrete-time sliding mode reaching condition.

\section{MAIN RESULTS}

In this section, the results of slidng surface design and robust DT-SMC law will be presented for a class of uncertain linear systems with time-varying delays. Let us first consider the problem of sliding surface design. In order to reduce the overdesign entailed in the stability analysis methods for timedelay systems, we employ the descriptor approach to derive the delay-dependent sufficient conditions for the existence of the sliding surface in terms of LMIs. The first result on the asymptotic stability of designing sliding surface is presented in the following theorem.

Theorem 1. If, for certain prescribed positive number $\kappa$, there exist positive scalars $\delta_{1}>0, \delta_{2}>0,(n-m) \times(n-m)$, matrices $L_{1}>0, L_{2}, L_{3}, U_{1}>0, U_{2}>0, \bar{W}_{1}, \bar{W}_{2}, \bar{W}_{3}$, and $m \times$ $(n-m)$ matrix $K$ such that the following LMIs hold:

$$
\begin{aligned}
& {\left[\begin{array}{cccccccc}
\Theta_{1} & \Theta_{2} & 0 & 0 & L_{1} & \vartheta & L_{2}^{\mathrm{T}} & L_{2}^{\mathrm{T}} \\
* & \Theta_{3} & \varsigma & 0 & 0 & 0 & L_{3}^{\mathrm{T}} & L_{3}^{\mathrm{T}} \\
* & * & -U_{1} & U_{1} H_{2}^{\mathrm{T}} & 0 & 0 & 0 & 0 \\
* & * & * & -\delta_{2} I & 0 & 0 & 0 & 0 \\
* & * & * & * & \chi & 0 & 0 & 0 \\
* & * & * & * & * & -\delta_{1} I & 0 & 0 \\
* & * & * & * & * & * & -L_{1} & 0 \\
* & * & * & * & * & * & * & -d_{M}^{-1} U_{2}
\end{array}\right]<0,} \\
& {\left[\begin{array}{ccc}
\bar{W}_{1} & \bar{W}_{2} & 0 \\
* & \bar{W}_{3} & \kappa A_{d} U_{1} \\
* & * & U_{1}
\end{array}\right] \geq 0,}
\end{aligned}
$$

where $\vartheta$ denotes $L_{1} H_{1}^{\mathrm{T}}-K^{\mathrm{T}} H_{3}^{\mathrm{T}}$, $\varsigma$ denotes $(1-\kappa) A_{d} U_{1}$, and $\chi$ denotes $-\left(d_{M}-d_{m}+1\right)^{-1} U_{1}$,

$$
\begin{aligned}
& \Theta_{1}=L_{2}+L_{2}^{\mathrm{T}}+d_{M} \bar{W}_{1}, \\
& \Theta_{2}=L_{3}+L_{1}\left(A^{\mathrm{T}}+\kappa A_{d}^{\mathrm{T}}-I\right)-K^{\mathrm{T}} A_{12}^{\mathrm{T}}-L_{2}^{\mathrm{T}}+d_{M} \bar{W}_{2}, \\
& \Theta_{3}=-L_{3}-L_{3}^{\mathrm{T}}+d_{M} \bar{W}_{3}+\sum_{i=1}^{2} \delta_{i} E_{i} E_{i}^{\mathrm{T}} .
\end{aligned}
$$

Then, the system (4) with Assumption 1 is asymptotically stable, and the sliding surface of (3) is given by

$$
S(k)=K L_{1}^{-1} x_{1}(k)+x_{2}(k)=0 .
$$

Proof. Let $\eta(k)=x_{1}(k+1)-x_{1}(k)$, the system (4) can be rewritten in an equivalent descriptor form:

$$
\begin{gathered}
x_{1}(k+1)=x_{1}(k)+\eta(k), \\
0=\left(\bar{A}_{11}(k)+\bar{A}_{d 11}(k)-I\right) x_{1}(k)-\eta(k)-\bar{A}_{d 11}(k) \sum_{i=k-d(k)}^{k-1} \eta(i),
\end{gathered}
$$

where

$$
\begin{gathered}
\bar{A}_{11}(k)=A_{11}-A_{12} C+\Delta A_{11}-\Delta A_{12} C, \\
\bar{A}_{d 11}(k)=A_{d 11}+\Delta A_{d 11} .
\end{gathered}
$$

Choose the Lyapunov-Krasovskii functional candidate as

$$
V(k)=V_{1}(k)+V_{2}(k)+V_{3}(k)+V_{4}(k) .
$$

Here,

$$
\begin{aligned}
& V_{1}(k)=x_{1}^{\mathrm{T}}(k) P_{1} x_{1}(k), \\
& V_{2}(k)=\sum_{i=k-d(k)}^{k-1} x_{1}^{\mathrm{T}}(i) Q x_{1}(i), \\
& V_{3}(k)=\sum_{j=-d_{M}+2} \sum_{i=k+j-1}^{-d_{m}+1} x_{1}^{\mathrm{T}}(i) Q x_{1}(i), \\
& V_{4}(k)=\sum_{j=d_{M}} \sum_{i=k+j}^{k-1} \eta^{\mathrm{T}}(i) G \eta(i),
\end{aligned}
$$

where $P_{1}, Q, G \in \mathbb{R}^{(n-m) \times(n-m)}$ are symmetric positive definite matrices to be determined. The LyapunovKrasovskii functional candidate $V(k)$ is positive definite for all $x_{1}(k) \neq 0$. Now, in order to evaluate the forward difference $\Delta V(k)=V(k+1)-V(k)$, in what follows we calculate $\Delta V_{1}, \Delta V_{2}, \Delta V_{3}$, and $\Delta V_{4}$, respectively. For $\Delta V_{1}(k)$, by using the descriptor system form (12), we obtain 
$\Delta V_{1}(k)$

$$
\begin{aligned}
= & \eta^{\mathrm{T}}(k) P_{1} \eta(k)+2 x_{1}^{\mathrm{T}}(k) P_{1} \eta(k) \\
= & \eta^{\mathrm{T}}(k) P_{1} \eta(k)+2 \bar{x}_{1}^{\mathrm{T}}(k) P^{\mathrm{T}}\left[\begin{array}{c}
\eta(k) \\
0
\end{array}\right] \\
= & \eta^{\mathrm{T}}(k) P_{1} \eta(k)+2 \bar{x}_{1}(k) P^{\mathrm{T}}\left[\begin{array}{c}
\eta(k) \\
\left(\bar{A}_{11}(k)+\bar{A}_{d 11}(k)-I\right) x_{1}(k)-\eta(k)
\end{array}\right] \\
& -2 \bar{x}_{1}^{\mathrm{T}}(k) P^{\mathrm{T}} \sum_{i=k-d(k)}^{k-1} K_{n-m} \bar{A}_{d 11}(k) \eta(i),
\end{aligned}
$$

where $\bar{x}_{1}^{\mathrm{T}}(k)=\left[x_{1}^{\mathrm{T}}(k) \eta^{\mathrm{T}}(k)\right], K_{n-m}=\left[\begin{array}{ll}0 & I\end{array}\right]^{\mathrm{T}}$, and $P=$ $\left[\begin{array}{cc}P_{1} & 0 \\ P_{2} & P_{3}\end{array}\right]$. If identifying $N:=P^{\mathrm{T}} K_{n-m} \bar{A}_{d 11}(k), a:=\bar{x}_{1}(k)$, and $b:=\eta(i)$ in (16), then applying Lemma 1 gives rise to the following inequality:

$\Delta V_{1}(k)$

$$
\begin{aligned}
& \leq \eta^{\mathrm{T}}(k) P_{1} \eta(k)+2 \bar{x}_{1}^{\mathrm{T}}(k) P^{\mathrm{T}} \\
& \times\left[\begin{array}{c}
\eta(k) \\
\left(\bar{A}_{11}(k)+\bar{A}_{d 11}(k)-I\right) x_{1}(k)-\eta(k)
\end{array}\right] \\
& +\sum_{i=k-d(k)}^{k-1}\left[\begin{array}{c}
\bar{x}_{1}(k) \\
\eta(i)
\end{array}\right]^{\mathrm{T}}\left[\begin{array}{cc}
W & \left.M-P^{\mathrm{T}} K_{n-m} \bar{A}_{d 11}(k)\right]\left[\begin{array}{c}
\bar{x}_{1}(k) \\
\eta
\end{array}\right. \\
\eta(i)
\end{array}\right] \\
& \leq \eta^{\mathrm{T}}(k) P_{1} \eta(k)+2 \bar{x}_{1}^{\mathrm{T}}(k) P^{\mathrm{T}}\left[\begin{array}{c}
\eta(k) \\
\left(\bar{A}_{11}(k)+\bar{A}_{d 11}(k)-I\right) x_{1}(k)-\eta(k)
\end{array}\right] \\
& +d_{M} \bar{x}_{1}^{\mathrm{T}}(k) W \bar{x}_{1}(k)+\sum_{i=k-d_{M}}^{k-1} \eta^{\mathrm{T}}(i) G \eta(i) \\
& +2 \bar{x}_{1}^{\mathrm{T}}(k)\left[M-P^{\mathrm{T}} K_{n-m} \bar{A}_{d 11}(k)\right]\left[x_{1}(k)-x_{1}(k-d(k))\right],
\end{aligned}
$$

where $W, M \in \mathbb{R}^{(n-m) \times(n-m)}$ are constant matrices with appropriate dimensions satisfying

$$
\left[\begin{array}{ll}
W & M \\
* & G
\end{array}\right] \geq 0 .
$$

For $\Delta V_{2}$, we have

$$
\begin{aligned}
\Delta V_{2}= & \sum_{i=k-d(k+1)+1}^{k} x_{1}^{\mathrm{T}}(i) Q x_{1}(i)-\sum_{i=k-d(k)}^{k-1} x_{1}^{\mathrm{T}}(i) Q x_{1}(i) \\
= & x_{1}^{\mathrm{T}}(k) Q x_{1}(k)-x_{1}^{\mathrm{T}}(k-d(k)) Q x_{1}(k-d(k)) \\
& +\sum_{i=k-d(k+1)+1}^{k-1} x_{1}^{\mathrm{T}}(i) Q x_{1}(i)-\sum_{i=k-d(k)+1}^{k-1} x_{1}^{\mathrm{T}}(i) Q x_{1}(i) \\
\leq & x_{1}^{\mathrm{T}}(k) Q x_{1}(k)-x_{1}^{\mathrm{T}}(k-d(k)) Q x_{1}(k-d(k)) \\
& +\sum_{i=k-d_{M}+1}^{k-d_{m}} x_{1}^{\mathrm{T}}(i) Q x_{1}(i) .
\end{aligned}
$$

For $\Delta V_{3}$, we get

$$
\begin{aligned}
\Delta V_{3} & =\sum_{j=-d_{M}+2}^{-d_{m}+1}\left[x_{1}^{\mathrm{T}}(k) Q x_{1}(k)-x_{1}^{\mathrm{T}}(k+j-1) Q x_{1}(k+j-1)\right] \\
& =\left(d_{M}-d_{m}\right) x_{1}^{\mathrm{T}}(k) Q x_{1}(k)-\sum_{j=k-d_{M}+1}^{k-d_{m}} x_{1}^{\mathrm{T}}(j) Q x_{1}(j) .
\end{aligned}
$$

For $\Delta V_{4}$, we obtain

$$
\begin{aligned}
\Delta V_{4} & =\sum_{j=-d_{M}}^{-1}\left[\eta^{\mathrm{T}}(k) G \eta(k)-\eta^{\mathrm{T}}(k+j) G \eta(k+j)\right] \\
& =d_{M} \eta^{\mathrm{T}}(k) G \eta(k)-\sum_{j=k-d_{M}}^{k-1} \eta^{\mathrm{T}}(j) G \eta(j) .
\end{aligned}
$$

Based on the above results in (17), (19)-(21), and using Lemma 2, we can derive

$$
\begin{aligned}
& \Delta V(k)=\Delta V_{1}(k)+\Delta V_{2}(k)+\Delta V_{3}(k)+\Delta V_{4}(k) \\
& \leq \bar{x}_{1}^{\mathrm{T}}(k) \Psi \bar{x}_{1}(k)+2 \bar{x}_{1}^{\mathrm{T}}(k) \\
& \times\left(P^{\mathrm{T}} K_{n-m} A_{d 11}-M\right) x_{1}(k-d(k)) \\
& +x_{1}^{\mathrm{T}}(k-d(k))\left(\delta_{2}^{-1} H_{2}^{\mathrm{T}} H_{2}-Q\right) x_{1}(k-d(k)) \\
& =\left[\begin{array}{lll}
x_{1}^{\mathrm{T}}(k) & \eta^{\mathrm{T}}(k) & x_{1}^{\mathrm{T}}(k-d(k))
\end{array}\right] \\
& \times\left[\begin{array}{cc}
\Psi & P^{\mathrm{T}}\left[\begin{array}{c}
0 \\
A_{d 11}
\end{array}\right]-M \\
* & \delta_{2}^{-1} H_{2}^{\mathrm{T}} H_{2}-Q
\end{array}\right]\left[\begin{array}{c}
x_{1}^{\mathrm{T}}(k) \\
\eta^{\mathrm{T}}(k) \\
x_{1}^{\mathrm{T}}(k-d(k))
\end{array}\right],
\end{aligned}
$$

where

$$
\begin{aligned}
\Psi= & P^{\mathrm{T}}\left[\begin{array}{cc}
0 & I \\
A_{11}-A_{12} C-I & -I
\end{array}\right] \\
& +\left[\begin{array}{cc}
0 & A_{11}^{\mathrm{T}}-C^{\mathrm{T}} A_{12}^{\mathrm{T}}-I \\
I & -I
\end{array}\right] P+d_{M} W+\left[\begin{array}{cc}
M & 0
\end{array}\right] \\
& +\left[\begin{array}{c}
M^{\mathrm{T}} \\
0
\end{array}\right]+\left[\begin{array}{cc}
\omega & 0 \\
0 & P_{1}+d_{M} G
\end{array}\right]+\sum_{i=1}^{2} \delta_{i} P^{\mathrm{T}}\left[\begin{array}{l}
0 \\
E_{i}
\end{array}\right]\left[\begin{array}{ll}
0 & E_{i}^{\mathrm{T}}
\end{array}\right] P
\end{aligned}
$$

where $\omega$ denotes $\delta_{1}^{-1}\left(H_{1}^{\mathrm{T}}-C^{\mathrm{T}} H_{3}^{\mathrm{T}}\right)\left(H_{1}-H_{3} C\right)+\left(d_{M}-\right.$ $\left.d_{m}+1\right) Q$ and $\delta_{1}, \delta_{2}$ are any positive scalars. Let $\xi=$ $\left[\begin{array}{lll}x_{1}^{\mathrm{T}}(k) & \eta^{\mathrm{T}}(k) & x_{1}^{\mathrm{T}}(k-d(k))\end{array}\right]$ and

$$
\Phi=\left[\begin{array}{cc}
\Psi & P^{\mathrm{T}}\left[\begin{array}{c}
0 \\
A_{d 11}
\end{array}\right]-M \\
* & \delta_{2}^{-1} H_{2}^{\mathrm{T}} H_{2}-Q
\end{array}\right] .
$$

Then

$$
\Delta V(k) \leq \xi^{\mathrm{T}}(k) \Phi \xi(k) .
$$


In order to obtain a convenient LMI, we restrict the choice of $M$ to be

$$
M=\kappa P^{\mathrm{T}}\left[\begin{array}{c}
0 \\
A_{d 11}(k)
\end{array}\right] .
$$

Define

$$
\begin{aligned}
& P^{-1}=L:=\left[\begin{array}{cc}
L_{1} & 0 \\
L_{2} & L_{3}
\end{array}\right], \quad K=C L_{1}, \quad Q^{-1}=U_{1}, \\
& G^{-1}=U_{2}, \quad \bar{W}=\left(P^{-1}\right)^{\mathrm{T}} W\left(P^{-1}\right)=\left[\begin{array}{ll}
\bar{W}_{1} & \bar{W}_{2} \\
\bar{W}_{2}^{\mathrm{T}} & \bar{W}_{3}
\end{array}\right] .
\end{aligned}
$$

Multiply (18) by $\operatorname{diag}\left[\left(P^{-1}\right)^{\mathrm{T}}, U_{2}\right]$ and $\operatorname{diag}\left[\left(P^{-1}\right), U_{2}\right]$ on the left- and right-hand sides, respectively. Alternatively, multiply (24) by $\operatorname{diag}\left[\left(P^{-1}\right)^{\mathrm{T}}, U_{1}\right]$ and $\operatorname{diag}\left[\left(P^{-1}\right), U_{1}\right]$ on the left- and right-hand sides, respectively. By using the Schur complement [19], we obtain that (8) is equivalent to $\Phi<0$, and (18) is equivalent to (9), which yields

$$
\Delta V(k)<0, \quad \forall \xi(k) \neq 0 .
$$

It follows from Lyapunov stability theory [20] that the system (4) is asymptotically stable. Moreover, based on the above definition of the sliding surface in (3), it can be obtained with $C=K L_{1}^{-1}$.

Once the sliding surface is appropriately designed according to Theorem 1, the next result on the design of DT$\mathrm{SMC}$ is summarized in the following theorem.

Theorem 2. Consider the system (2) with Assumptions 1-3. If the linear sliding surface is designed as in (11), then the following DT-SMC law

$$
\begin{gathered}
u(k)=-B^{-1}\left\{\sum_{i=1}^{2}\left[\left(A_{2 i}+C A_{1 i}\right) x_{i}(k)\right]-(1-q \tau) S(k)\right. \\
+\epsilon \tau \cdot \operatorname{sgn}(S(k))+\left(\alpha_{0}+\beta_{0}+\gamma_{0}\right) \\
\left.+\left(\rho_{\alpha}+\rho_{\beta}+\rho_{\gamma}\right) \cdot \operatorname{sgn}(S(k))\right\}
\end{gathered}
$$

can guarantee the discrete-time sliding mode reaching condition. Here,

$$
\begin{aligned}
S(k) & =\left[\begin{array}{llll}
S_{1}(k) & S_{2}(k) & \cdots & S_{m}(k)
\end{array}\right], \\
\operatorname{sgn}(S(k)) & =\left[\begin{array}{llll}
\operatorname{sgn}\left(S_{1}\right) & \operatorname{sgn}\left(S_{2}\right) & \cdots & \operatorname{sgn}\left(S_{m}\right)
\end{array}\right], \\
\alpha_{0} & =\frac{\alpha_{U}+\alpha_{L}}{2}, \quad \beta_{0}=\frac{\beta_{U}+\beta_{L}}{2}, \quad \gamma_{0}=\frac{\gamma_{U}+\gamma_{L}}{2}, \\
\rho_{\alpha} & =\frac{\alpha_{U}-\alpha_{L}}{2}, \quad \rho_{\beta}=\frac{\beta_{U}-\beta_{L}}{2}, \quad \rho_{\gamma}=\frac{\gamma_{U}-\gamma_{L}}{2},
\end{aligned}
$$

$C=K L_{1}^{-1}, \alpha_{U}$ and $\alpha_{L}$ are the upper bound and lower bound of $\widetilde{\alpha}(k)=C \Delta A_{11} x_{1}(k)+C \Delta A_{12} x_{2}(k), \beta_{U}$ and $\beta_{L}$ are the upper bound and lower bound of $\tilde{\beta}(k)=C \Delta A_{d 11} x_{1}(k-d(k))+$ $C A_{d 11} x_{1}(k-d(k))+\sum_{i=1}^{2}\left[A_{d 2 i} x_{i}(k-d(k))\right], \gamma_{U}$ and $\gamma_{L}$ are the upper bound and lower bound of $\tilde{\gamma}(k)=f\left(k, x_{k}\right), q>0$, $\varepsilon>0,1-q \tau>0$, and $\tau>0$ is the sampling period.

Proof. The design of DT-SMC law guarantees that the reaching condition must be satisfied when there exist uncertainties and time-varying delays in systems. We consider the reaching condition for the DT-SMC proposed in [21] as follows:

$$
\begin{aligned}
\Delta S(k) & =S(k+1)-S(k) \\
& \leq-\epsilon \tau \cdot \operatorname{sgn}(S(k))-q \tau S(k), \quad \text { if } S(k)>0, \\
\Delta S(k) & =S(k+1)-S(k) \\
& \geq-\epsilon \tau \cdot \operatorname{sgn}(S(k))-q \tau S(k), \quad \text { if } S(k)<0 .
\end{aligned}
$$

From the designed sliding surface

$$
S(k)=\bar{C} x(k)=[C I] x(k),
$$

we have

$$
\begin{aligned}
\Delta S(k)= & \left(C A_{11}+C \Delta A_{11}\right) x_{1}(k)+\left(C A_{d 11}+C \Delta A_{d 11}\right) x_{1}(k-d(k)) \\
& +\left(C A_{12}+C \Delta A_{12}\right) x_{2}(k) \\
& +\sum_{i=1}^{2}\left[A_{2 i} x_{i}(k)+A_{d 2 i} x_{i}(k-d(k))\right]+B u(k)+f\left(k, x_{k}\right) .
\end{aligned}
$$

Since it is assumed that $\Delta A_{11}, \Delta A_{d 11}, \Delta A_{12}$, and $f\left(k, x_{k}\right)$ are bounded, using the discrete-time version of improved Razumikhin theorem in [22], for any solution $x(k-d(k))$ of (2), there exists a constant $\theta$ such that the following inequality is satisfied:

$$
\|x(k-d(k))\|<\theta\|x(k)\|, \quad \forall k, d_{m} \leq d(k) \leq d_{M} .
$$

We can also obtain that $\tilde{\alpha}(k)=C \Delta A_{11} x_{1}(k)+C \Delta A_{12} x_{2}(k)$, $\tilde{\beta}(k)=C\left(A_{d 11}+\Delta A_{d 11}\right) x_{1}(k-d(k))+\sum_{i=1}^{2}\left[A_{d 2 i} x_{i}(k-d(k))\right]$, and $\tilde{\gamma}(k)=f\left(k, x_{k}\right)$ will be bounded with upper and lower bounds. Let the bounds be

$$
\alpha_{L} \leq \tilde{\alpha}(k)=C \Delta A_{11} x_{1}(k)+C \Delta A_{12} x_{2}(k) \leq \alpha_{U},
$$

$$
\begin{aligned}
& \beta_{L} \leq \tilde{\beta}(k) \\
& =C\left(A_{d 11}+\Delta A_{d 11}\right) x_{1}(k-d(k))+\sum_{i=1}^{2}\left[A_{d 2 i} x_{i}(k-d(k))\right] \leq \beta_{U}, \\
& \gamma_{L} \leq \tilde{\gamma}=f\left(k, x_{k}\right) \leq \gamma_{U} .
\end{aligned}
$$

Here, the inequality

$$
\begin{aligned}
\alpha_{L} & =\left[\begin{array}{llll}
\alpha_{L, 1} & \alpha_{L, 2} & \cdots & \alpha_{L, m}
\end{array}\right]^{\mathrm{T}} \\
& \leq \widetilde{\alpha}(k)=\left[\begin{array}{llll}
\tilde{\alpha}_{1} & \tilde{\alpha}_{2} & \cdots & \tilde{\alpha}_{m}
\end{array}\right]^{\mathrm{T}} \\
& \leq \alpha_{U}=\left[\begin{array}{llll}
\alpha_{U, 1} & \alpha_{U, 2} & \cdots & \alpha_{U, m}
\end{array}\right]^{\mathrm{T}},
\end{aligned}
$$

implies that $\alpha_{L, i} \leq \tilde{\alpha}_{i}(k) \leq \alpha_{U, i}, i=1,2, \ldots, m$; similar notations are used for $\tilde{\beta}(k)$ and $\tilde{\gamma}(k)$. 
Then, substituting (29) into (33), we have

$$
\begin{aligned}
\Delta S(k)= & \tilde{\alpha}(k)+\tilde{\beta}(k)+\tilde{\gamma}(k)-q \tau S(k)-\epsilon \tau \cdot \operatorname{sgn}(S(k)) \\
& -\left(\alpha_{0}+\beta_{0}+\gamma_{0}\right)-\left(\rho_{\alpha}+\rho_{\beta}+\rho_{\gamma}\right) \cdot \operatorname{sgn}(S(k)) .
\end{aligned}
$$

According to previous discussion, the following relations hold:

$$
\begin{array}{ll}
\tilde{\alpha}(k) \leq \alpha_{0}+\rho_{\alpha} \cdot \operatorname{sgn}(S(k)) & \text { if } S(k)>0, \\
\tilde{\alpha}(k) \geq \alpha_{0}+\rho_{\alpha} \cdot \operatorname{sgn}(S(k)) & \text { if } S(k)<0, \\
\tilde{\beta}(k) \leq \beta_{0}+\rho_{\beta} \cdot \operatorname{sgn}(S(k)) & \text { if } S(k)>0, \\
\tilde{\beta}(k) \geq \beta_{0}+\rho_{\beta} \cdot \operatorname{sgn}(S(k)) & \text { if } S(k)<0, \\
\tilde{\gamma}(k) \leq \gamma_{0}+\rho_{\gamma} \cdot \operatorname{sgn}(S(k)) & \text { if } S(k)>0, \\
\tilde{\gamma}(k) \geq \gamma_{0}+\rho_{\gamma} \cdot \operatorname{sgn}(S(k)) & \text { if } S(k)<0 .
\end{array}
$$

Thus, the sign change of $\Delta S(k)$ in (37) is opposite to that of $S(k)$, irrespective of the value of the uncertainties $\tilde{\alpha}(k)$, $\tilde{\beta}(k)$, and $\tilde{\gamma}(k)$. Moreover, the closed-loop control system (2) satisfies the reaching condition (31).

Remark 1. The descriptor system approach $[13,14,16]$ can greatly reduce the overdesign entailed in the existing methods because the descriptor model leads to a system that is equivalent to the original one (from the point of view of stability) and requires bounding of fewer cross-terms. Therefore, in this work, we apply the descriptor system approach to the SMC design for discrete-time systems with uncertainties and time-varying delays.

Remark 2. SMC approach is an effective method to achieve the robustness and invariant property to matched uncertainties and disturbance on the sliding surface $[9,21]$. In this paper, the results of Theorem 1 guarantee that the sliding motion dynamics will be robustly asymptotically stable. Furthermore, the descriptor-system-approach-based DT-SMC in Theorem 2 provides a solution to satisfy the sliding mode reaching condition of the designed discrete-time sliding surface for discrete-time systems with uncertainties and time-varying delays.

\section{NUMERICAL EXAMPLE}

In this section, an illustrative example is given to verify the design method proposed in this paper. Consider the discretetime system (2) with the following description:

$$
\begin{aligned}
x_{1}(k) & =\left[\begin{array}{l}
x_{11}(k) \\
x_{12}(k)
\end{array}\right], \quad A_{11}=\left[\begin{array}{cc}
0.7 & 0 \\
0.05 & 0.8
\end{array}\right], \\
A_{d 11} & =\left[\begin{array}{cc}
-0.2 & 0 \\
0 & -0.1
\end{array}\right], \quad A_{12}=\left[\begin{array}{c}
0.3 \\
0
\end{array}\right], \\
A_{21} & =\left[\begin{array}{c}
-0.5 \\
0
\end{array}\right], \quad A_{22}=2.0,
\end{aligned}
$$

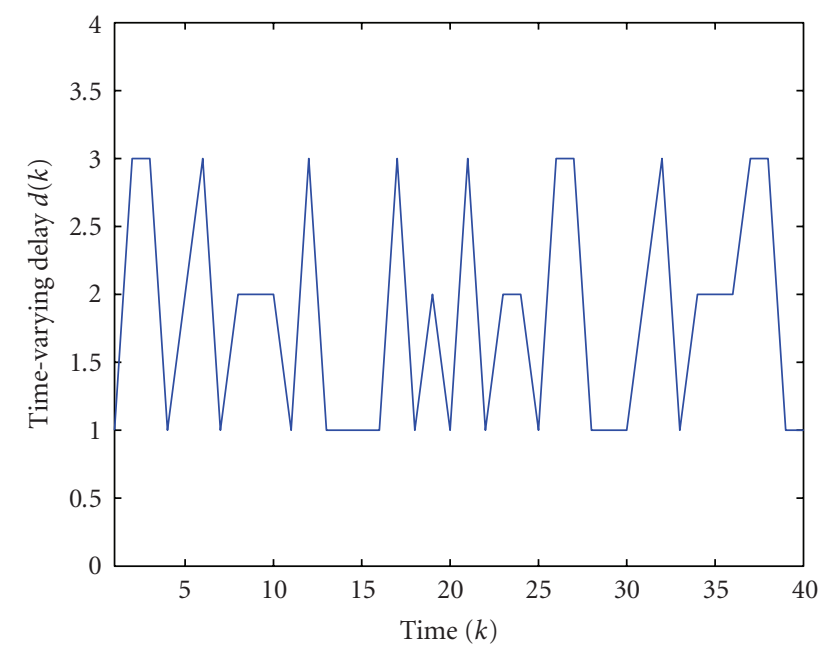

FIGURE 1: Time-varying delays $d(k)$.

$$
\begin{aligned}
A_{d 21} & =\left[\begin{array}{c}
0 \\
0.1
\end{array}\right], \quad A_{d 22}=0.05, \quad B=1, \\
\Delta A_{11} & =\left[\begin{array}{ll}
0.02 \sin (0.01 k \pi) & 0.02 \sin (0.01 k \pi) \\
0.01 \cos (0.01 k \pi) & 0.04 \sin (0.01 k \pi)
\end{array}\right], \\
\Delta A_{12} & =\left[\begin{array}{l}
0.05 \\
0.04
\end{array}\right], \\
\Delta A_{d 11} & =\left[\begin{array}{ll}
0.01 \sin (0.01 k \pi) & 0.04 \sin (0.01 k \pi) \\
0.01 \cos (0.01 k \pi) & 0.05 \sin (0.01 k \pi)
\end{array}\right], \\
f(k, x(k))= & 0.4 \sin \left(x_{11}(k)\right) .
\end{aligned}
$$

Initial states of the system are $x_{1}(k)=\left[\begin{array}{ll}1.0 & -0.5\end{array}\right]^{\mathrm{T}}, x_{2}(k)=$ 0.6 , for $k \in\left[-d_{M} 0\right]$. Using Theorem 1 and choosing $\kappa=$ 0.5 , we obtain $C=\left[\begin{array}{ll}0.9305 & 0.5168\end{array}\right]$. Then, the linear sliding surface is $S(k)=\left[\begin{array}{ll}C & 1\end{array}\right] x(k)$. According to Theorem 2, the robust DT-SMC law is designed as

$$
\begin{array}{r}
u(k)=-B^{-1}\left\{\sum_{i=1}^{2}\left[\left(A_{2 i}+C A_{1 i}\right) x_{i}(k)\right]-(1-q \tau) S(k)\right. \\
\left.+\left(\epsilon \tau+\rho_{\alpha}+\rho_{\beta}+\rho_{\gamma}\right) \cdot \operatorname{sgn}(S(k))\right\},
\end{array}
$$

where $\rho_{\alpha}=0.0238\left|x_{11}(k)\right|+0.0393\left|x_{12}(k)\right|+0.0672\left|x_{2}(k)\right|$, $\rho_{\beta}=0.5014\left|x_{11}(k)\right|+0.2785\left|x_{12}(k)\right|+0.125\left|x_{2}(k)\right|, \rho_{\gamma}=$ $0.4\left|\sin x_{11}(k)\right|, \tau=0.1, q=5$, and $\epsilon=0.1$. The timevarying delay $1 \leq d(k) \leq 3$ is shown in Figure 1 . System state trajectories are illustrated in Figure 2. The resulting sliding surface is in Figure 3. Figure 4 depicts the control input signal.

It is observed from Figure 2 that the state trajectories of the system all converge to the origin quickly. The system can be stabilized quickly by the proposed method and the reaching motion satisfies the sliding reaching condition in spite of 


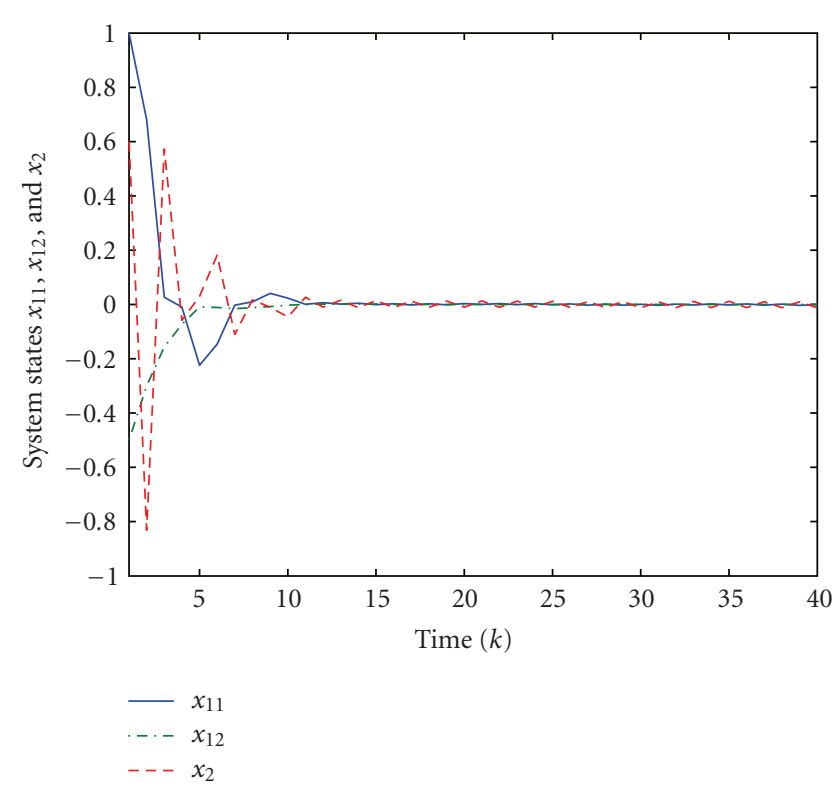

FIgURE 2: System states $x_{11}, x_{12}$, and $x_{2}$.

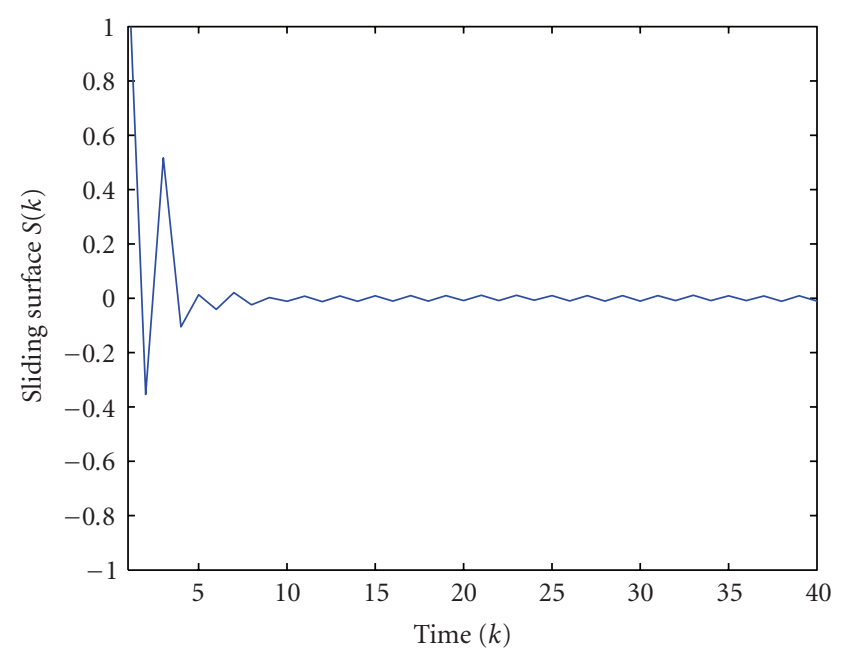

FIgURE 3: Sliding surface $S(k)$.

the time-varying delays and uncertainties. Simulation results illustrate that the proposed approach in this paper is feasible and effective for discrete-time uncertain linear systems with time-varying delays.

\section{CONCLUSION}

In this paper, the problem of robust DT-SMC for uncertain systems with time-varying delays has been studied. By using the descriptor model transformation and a recent result on bounding cross products of vectors, a delay-dependent sufficient condition for the existence of stable sliding surfaces is constructed for all admissible uncertainties. Based on this existence condition, the corresponding reaching motion controller is developed such that the reaching motion satisfies the discrete-time sliding mode reaching condition

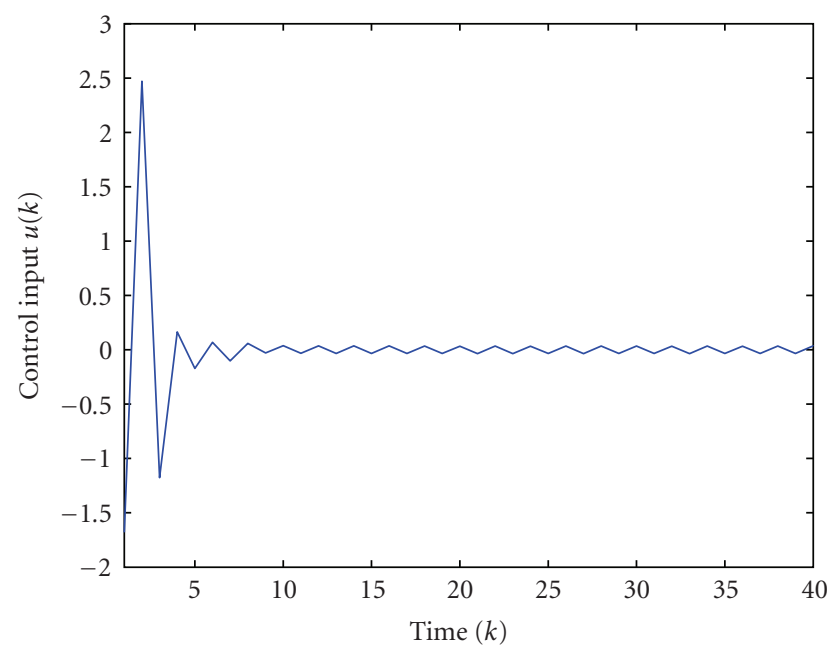

Figure 4: Control input $u(k)$.

for uncertain systems with time-varying delays. An example shows the validity and effectiveness of the proposed DT-SMC design method.

\section{ACKNOWLEDGMENTS}

The authors wish to thank the associate editor and anonymous reviewers for providing constructive suggestions which have improved the presentation of the paper. This research was supported by the Natural Sciences and Engineering Research Council of Canada and the Canada Foundation of Innovation.

\section{REFERENCES}

[1] E.-K. Boukas and Z.-K. Liu, Deterministic and Stochastic Time Delay Systems, Birkhäuser, Boston, Mass, USA, 2002.

[2] K. Gu, V. L. Kharitonov, and J. Chen, Stability of Time-Delay Systems, Birkhäuser, Boston, Mass, USA, 2003.

[3] M. Wu, Y. He, J.-H. She, and G.-P. Liu, "Delay-dependent criteria for robust stability of time-varying delay systems," Automatica, vol. 40, no. 8, pp. 1435-1439, 2004.

[4] P. Park and J. W. Ko, "Stability and robust stability for systems with a time-varying delay," Automatica, vol. 43, no. 10, pp. 1855-1858, 2007.

[5] W.-H. Chen, Z.-H. Guan, and X. Lu, "Delay-dependent guaranteed cost control for uncertain discrete-time systems with delay," IEE Proceedings: Control Theory and Applications, vol. 150, no. 4, pp. 412-416, 2003.

[6] L. Zhang, Y. Shi, T. Chen, and B. Huang, "A new method for stabilization of networked control systems with random delays," IEEE Transactions on Automatic Control, vol. 50, no. 8, pp. 1177-1181, 2005.

[7] H. Gao, J. Lam, C. Wang, and Y. Wang, "Delay-dependent output-feedback stabilization of discrete-time systems with time-varying state delay," IEE Proceedings: Control Theory and Applications, vol. 151, no. 6, pp. 691-698, 2004.

[8] H. Gao and T. Chen, "New results on stability of discrete-time systems with time-varying state delay," IEEE Transactions on Automatic Control, vol. 52, no. 2, pp. 328-334, 2007. 
[9] V. Utkin, "Variable structure systems with sliding modes," IEEE Transactions on Automatic Control, vol. 22, no. 2, pp. 212-222, 1977.

[10] A. J. Koshkouei and A. S. I. Zinober, "Sliding mode time delay systems," in Proceedings of the IEEE International Workshop on Variable Structure Systems (VSS '96), pp. 97-101, Tokyo, Japan, December 1996.

[11] Y. Xia and Y. Jia, "Robust sliding-mode control for uncertain time-delay systems: an LMI approach," IEEE Transactions on Automatic Control, vol. 48, no. 6, pp. 1086-1092, 2003.

[12] Y. Niu, J. Lam, X. Wang, and D. W. C. Ho, "Observer-based sliding mode control for nonlinear state-delayed systems," International Journal of Systems Science, vol. 35, no. 2, pp. 139150, 2004.

[13] E. Fridman, "New Lyapunov-Krasovskii functionals for stability of linear retarded and neutral type systems," Systems \& Control Letters, vol. 43, no. 4, pp. 309-319, 2001.

[14] E. Fridman and U. Shaked, "A descriptor system approach to $H_{\infty}$ control of linear time-delay systems," IEEE Transactions on Automatic Control, vol. 47, no. 2, pp. 253-270, 2002.

[15] E. Fridman and U. Shaked, "A new $H_{\infty}$ filter design for linear time delay systems," IEEE Transactions on Signal Processing, vol. 49, no. 11, pp. 2839-2843, 2001.

[16] E. Fridman, F. Gouaisbaut, M. Dambrine, and J.-P. Richard, "Sliding mode control of systems with time-varying delays via descriptor approach," International Journal of Systems Science, vol. 34, no. 8-9, pp. 553-559, 2003.

[17] Y. S. Moon, P. G. Park, W. H. Kwon, and Y. S. Lee, "Delaydependent robust stabilization of uncertain state-delayed systems," International Journal of Control, vol. 74, no. 14, pp. 1447-1455, 2001.

[18] L. Xie, "Output feedback control of systems with parameter uncertainty," International Journal of Control, vol. 63, no. 4, pp. 741-750, 1996.

[19] S. Boyd, L. E. Ghaoui, E. Feron, and V. Balakrishnan, Linear Matrix Inequalities in System and Control Theory, SIAM, Philadelphia, Pa, USA, 1994.

[20] M. Vidyasagar, Nonlinear Systems Analysis, Prentice-Hall, Upper Saddle River, NJ, USA, 1993.

[21] W. Gao, Y. Wang, and A. Homaifa, "Discrete-time variable structure control systems," IEEE Transactions on Industrial Electronics, vol. 42, no. 2, pp. 117-122, 1995.

[22] J. K. Hale and S. M. V. Lunel, Introduction to Functional Differential Equations, Springer, New York, NY, USA, 1993. 

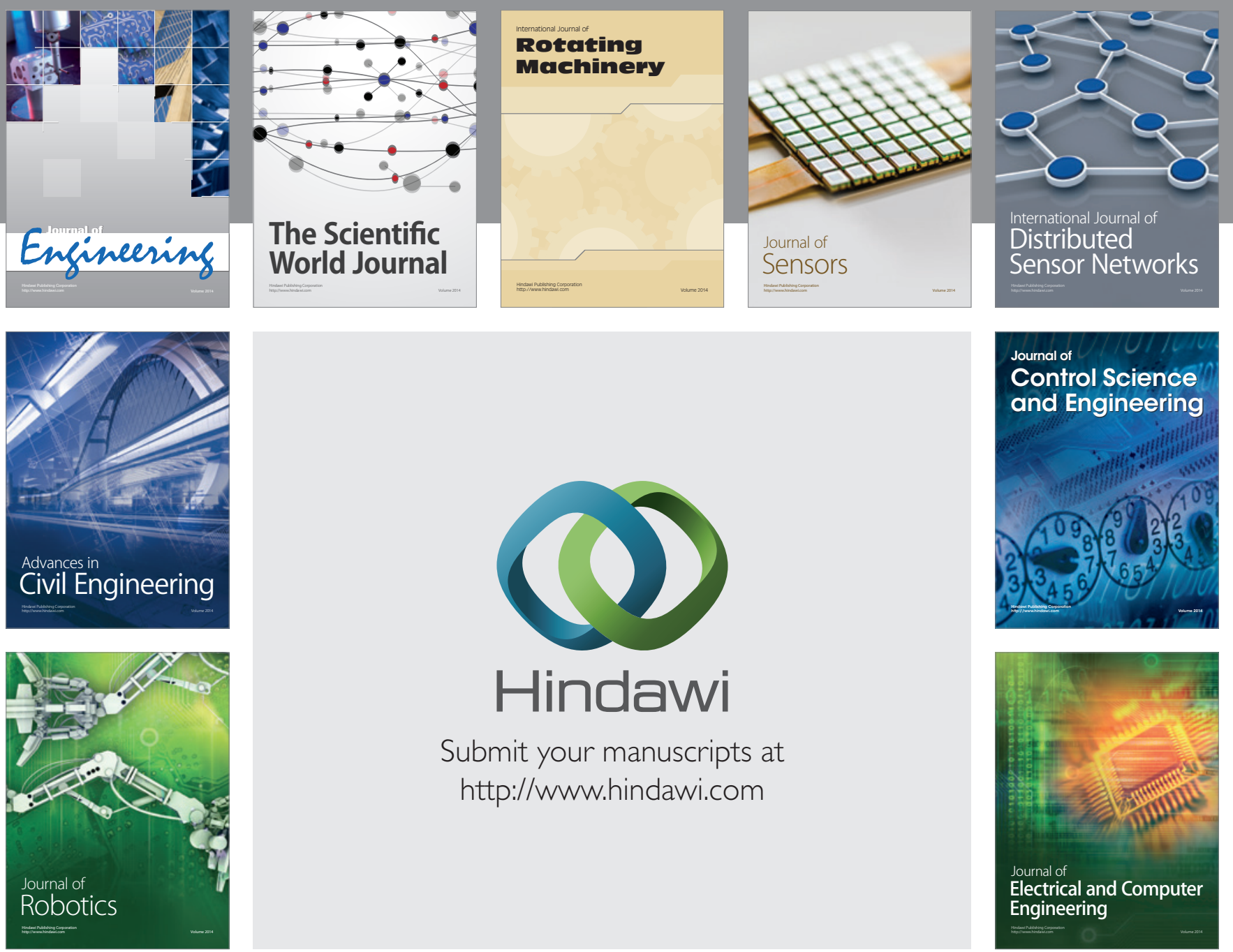

Submit your manuscripts at

http://www.hindawi.com
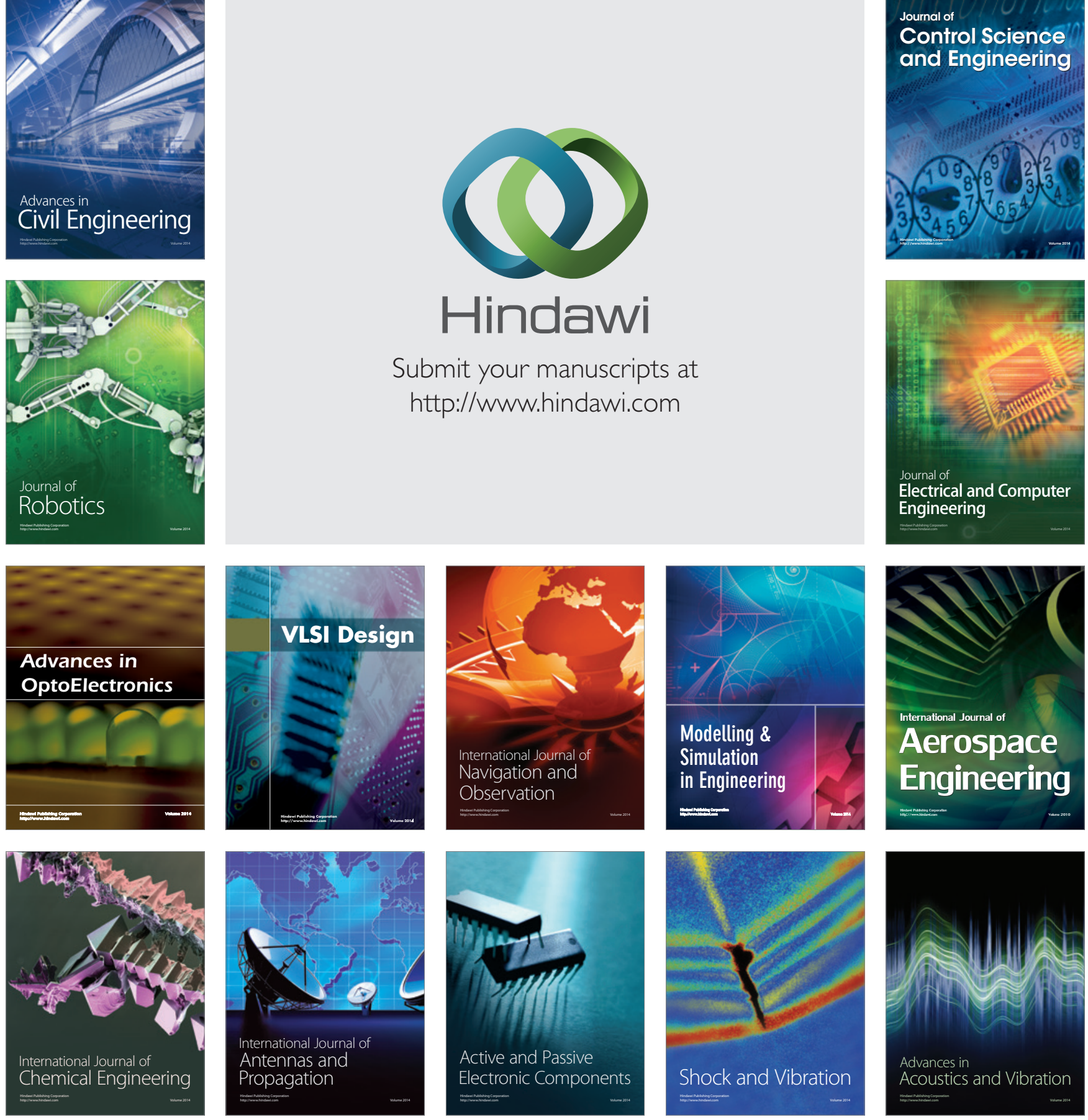